Review Articles

\title{
Melatonin, A Promising Therapeutic for Diabetes Mellitus and Its Complications: A Narrative Review
}

\author{
Yanuarita Tursinawati ${ }^{1}$, Arum Kartikadewi, ${ }^{1}$ R. Susanti, ${ }^{2}$ Ari Yuniastuti ${ }^{2 *}$ \\ ${ }^{1}$ Faculty of Medicine, University of Muhammadiyah Semarang, Indonesia \\ ${ }^{2}$ Faculty of Mathematics and Natural Sciences, Universitas Negeri Semarang, Indonesia
}

\section{Article Info}

History

Received: 08 Sept 2021

Accepted: 25 Dec 2021

Available: 31 Dec 2021

\begin{abstract}
Diabetes mellitus (DM) is a non-communicable disease and still a health problem in the world. Melatonin is responsible to metabolize carbohydrate and regulate blood sugar levels. Several studies proved Melatonin is useful in the diabetes therapy and its complication by performing antioxidant activity through various signaling pathways. The role of the melatonin hormone in the pathogenesis of complications of diabetes mellitus is still a lot of controversy. Animal studies are still incomplete to explain the mechanism of action of melatonin. Therefore, this review aims to discuss Melatonin role administration in DM and its complications. This review was started by searching articles using electronic databases such as Google Scholar, Science Direct, SpringerLink and Pubmed between 2009-2019. Melatonin increased respiration within mitochondria; acted as antioxidant that fight free radical production; and reduced oxidative stress. It diminished proinflammatory cytokines in diabetes and prevents various DM complications on eyes, cardiac and male reproductive organs through antioxidant effects. It inhibited proinflammatory within several signaling Pathways, included PI3K/Akt-Nrf2,cGMP-PKG, Nrf-2-HO-1 and MAPK, Syk, p38MAPK pathways. There are various melatonin roles in diabetes and DM complications as a promising new breakthrough of DM treatment. It is necessary to explore Melatonin role in other DM complications. Most of this research is on experimental animals, thus clinical trial studies should be carried out in humans to make melatonin a promising therapeutic or prevention for diabetes and its complications in the future.
\end{abstract}

Keyword: Melatonin; Diabetes Mellitus; Complication; Signaling Pathways Permalink/ DOI: https://doi.org/10.14710/jbtr.v7i3.12257

\section{INTRODUCTION}

High sugar level or hyperglycemia may be caused by cellular insensitivity to insulin in body, called as type 2 $\mathrm{DM}$ (T2DM). DM is one of the non-communicable diseases (NCDs) and is still health problem in the world with increasing morbidity and mortality incidence. In 2013, WHO targeted the prevention and control programs for NCDs until 2025, successfully reduced $25 \%$ mortality incidence. ${ }^{1}$ According to 2013 Basic Health Research data, DM incidence in Indonesia increased from $5.7 \%$ to $6.9 \%$ of total Indonesian population, 250 million people from 2007 to 2013 . $^{2}$

\footnotetext{
* Corresponding author:

E-mail: ariyuniastuti@mail.unnes.ac.id

(Ari Yuniastuti)
}

Health Profile of Central Java Province in 2017 noted that DM was the second highest non-communicable diseases with $19.22 \%$ after hypertension in Indonesia, becoming a priority for both the world and Indonesia to control due to its further complications. ${ }^{3}$

T2DM patients experience abnormalities of hormones to metabolize carbohydrate and regulate blood sugar levels, one of them is melatonin. The decreasing melatonin levels and increasing insulin level in Goto Kakizaki rat which is a T2DM experimental animal model were found. ${ }^{4}$ Melatonin is an indolamine hormone synthesized from amino acids L-tryptophan, especially in pineal gland and some extra pineal tissues such as gastrointestinal and lymphocyte.

Melatonin interacts with receptors in carrying out its functions. Two types of melatonin receptors humans are 
MT1 and MT2. MT1 is found in human pancreatic alpha cells. $^{5}$

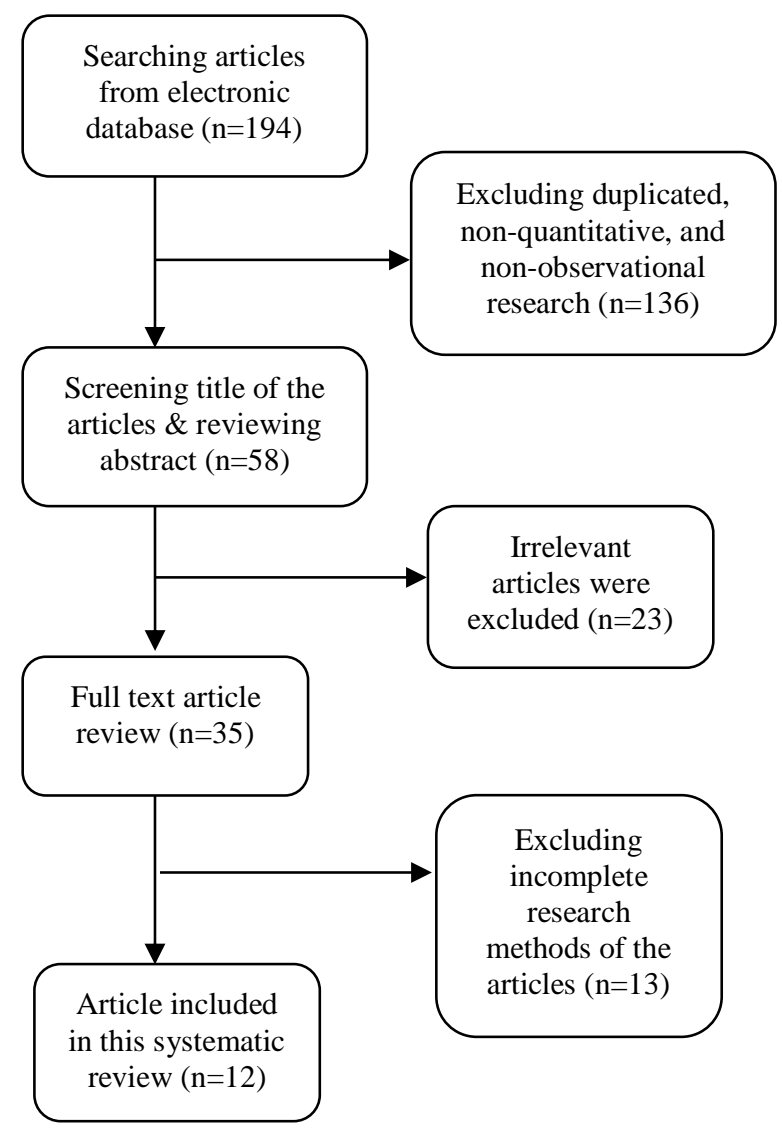

Figure 1. Article selection flowchart of literature searching process

Melatonin regulates insulin secretion by pancreatic beta cells both in vitro and in vivo, involving signals of intracellular transduction such as cAMP, cGMP and IP $3{ }^{6,7}$ The effect of the action of the hormone melatonin on insulin secretion in pancreatic beta cells, as well as its effect on target organs in the periphery, is thought to be through several mechanisms. Provision of melatonin increases abnormalities found in DM through action mechanism on mitochondria fat tissues and inflammation process. ${ }^{8,9}$

Uncontrolled blood sugar levels in DM patients causes complications for DM patients. Complications attack various organs such as the heart, brain, kidney, and eye. Studies have proven giving melatonin as an antioxidant to DM complications. Melatonin is a free radical scavenger to reactive oxygen species (ROS) and reactive nitrogen species (RNS), counteracting effectively oxidative stress to cause DMCs. Melatonin antioxidant effect appears due to its ability to activate glutathione, a cellular antioxidant. This mechanism is used to prevent or reduce progression of DM complications, for example in diabetic cataracts, and diabetic retinopathy. ${ }^{10,11}$ Role of antioxidant melatonin also involves a variety of cellular pathways to mediate in reducing incidence of cardiac complications: myocardial ischemia-reperfusion (MI/R) injury, myocardial contractile dysfunction, diabetic cardiomyopathy, and male reproductive organserectile dysfunction. ${ }^{12-16}$ In this review, it aims to discuss melatonin administration (MA) role in DM incidents and DM complications.

\section{MATERIALS AND METHODS}

Articles were searched through electronic databases such as Google Scholar, Science Direct, SpringerLink and Pubmed between 2009-2019, This systematic review used keywords: "DM, Diabetes Mellitus, Type 2 Diabetes Mellitus, Type 2 DM, type 2 diabetes, T2DM, Diabetic Patients, Non-Insulin Dependent Diabetes Mellitus, NIDDM, Insulin resistance, Nephropathy diabetes, diabetic nephropathy, Retinopathy diabetes, diabetic retinopathy, Neuropathy diabetes, diabetic neuropathy, Diabetic Foot ulcer, Diabetic Erectile dysfunction, Diabetic cardiovascular problem, Glucose intolerance, Macrovascular complication, Microvascular complication, Melatonin hormone, Melatonin, Melatonin receptor, Circadian rhythm".

The inclusive criteria were quantitative research, original articles with observational research designs-both animals and humans from 2009-2019, and full text accessible resulting twelve remaining articles (Figure 1). There was only one publication from twelve used human subject. The analysis focuses on melatonin role in DM and DMC associated to melatonin work to review articles. The articles were identified, filtered, assessed their feasibility, and extracted the data into a table with a predetermined title. Any discrepancy is discussed by authors.

\section{RESULTS}

Melatonin is a synthesized indolamine hormone from amino acids L-tryptophan, especially in pineal gland and some extra pineal tissues such as gastrointestinal and lymphocytes. ${ }^{17}$ Melatonin biosynthesis is triggered by releasing norepinephrine from sympathetic axons during dark conditions and started from melatonin precursor conversion (tryptophan) into 5-hydroxytryptophan by the aid of the tryptophan hydroxylase enzyme. Then 5hydroxithriptofan is decarboxylated to serotonin by the enzyme 5-hydroxithriptofan decarboxylase. Melatonin has antioxidant and is free radical scavenger to reactive oxygen species (ROS) and reactive nitrogen species (RNS) to counteract oxidative stress.

\section{Melatonin Role in Diabetes Mellitus}

T2DM patients suffered glucose homeostasis abnormalities due to degression in pancreatic beta cell function and insulin secretion. ${ }^{18}$ One of the hormones having role to metabolize carbohydrate and regulate blood glucose levels is melatonin. Melatonin regulates insulin secretion by pancreatic beta cells both in vitro and in vivo involving signals of intracellular transduction such as cAMP, GMP and IP3 signal pathway. ${ }^{6}$

A new theory addressing melatonin therapy for DM highlights its role as an antioxidant. Jimenéz et al, 2014 showed presence of mitochondrial dysfunction in obesity and T2DM patients. Mitochondrial dysfunction reduced ATP production in oxidative respiration, interfered with ROS or RNS formation.. ${ }^{8,19}$ Melatonin increased mitochondria respiration, fought free radical production, and reduced oxidative stress in various tissues such as the brain, liver, heart and skeletal muscles. ${ }^{20-22}$ This research identified melatonin role in fat tissue mitochondrial of 
diabetic mice. Mitochondrial dysfunction in fat tissue causes an increase in oxidative stress, apoptosis and inflammation resulting in insulin resistance. The results showed obese and diabetic rats had a significant increase in nitrite, whereas the amount of nitrite in lean rats and diabetic rats (ZDF) given by melatonin therapy was diminished.

Melatonin roles as an antioxidant was investigated by Kdziora et al,2009 that analyzed the effects of given melatonin to oxidative stress in humans. Theoretically, diabetes increases formation of ROS due to hyperglycemia, spurring glucose autooxidation, activation of sorbitol pathways, and impairing antioxidant enzyme activity. ${ }^{23}$ The parameters included malondialdehyde concentration (MDA), superoxide dismutase (SOD), morning melatonin concentration, and oxidase activity of ceruloplasmin (Cp). T2DM respondents were given melatonin $5 \mathrm{mg}, 1$ hour before going to bed for 30 days. The findings showed T2DM group had higher erythrocyte MDA level entailed by degression of erythrocyte SOD activity, proving lower antioxidant activity. Increased MDA levels indicating lipid peroxidation process occurred in diabetic conditions. Lipid peroxidation reduced membrane fluidity; increased nonspecific permeability; and deactivated several membrane enzymes leading to worse diabetes development. ${ }^{24}$ Morning melatonin levels were found lower on T2DM group. But after 30 days, morning melatonin levels increased significantly.

Another theory underlying pathophysiology of DM is increasing proinflammatory cytokines such as tumor necrosis factor- $\alpha$ (TNF- $\alpha$ ) and interleukin $1 \beta$ (IL-1 $\beta$ ), inducing damage to beta pancreas islet cells. ${ }^{25} \mathrm{DM}$ is a result of immune responses to dysregulation. Melatonin is considered being capable of inhibiting production of proinflammatory cytokines. Ozkaniar et al, 2016 identified effect of giving melatonin to inflammatory parameters and system immune responses of Diabetic rats. The results found that in DM group, TNF- $\alpha$ and IL$1 \beta$ levels were significantly increased compared to the control group. IL-1 $\beta$ levels in the DM group given melatonin were lower although it was not significant as well as TNF- $\alpha$ levels. TNF- $\alpha$ produced in adipocytes triggers insulin resistance by inhibiting tyrosine kinase receptor activity. The DM rats group had higher neutrophil levels. Neutrophils are markers of inflammatory response and indicators of injury stimulation. White blood cell (WBC), monocyte and neutrophil levels decreased significantly in the DM-Mel group. The exact mechanism of melatonin that reduced WBC has not been clearly explained, but there is a theory that melatonin contributes in reducing hematopoiesis process of bone marrow or lymphoid organs. ${ }^{26}$ Another theory suggests antioxidant effects of melatonin having role in hematopoietic system, causing a degression WBC. ${ }^{27}$ In conclusion, melatonin might help controlling immune system regulation through inhibition of proinflammatory production, inhibiting destruction of lymphoid organs.

Diabetes mellitus with insulin resistance is often followed by other conditions such as obesity, fat abnormalities, hypertension, which is known as the metabolic syndrome. Apparently, treatment of melatonin also has an effect on improving the status of patients with metabolic syndrome. Study by Bahrami et al, 2019 with randomized clinical trial among 70 metabolic syndrome patients showed a significant improvement in waist circumference, systolic blood pressure (SBP), diastolic blood pressure (DBP), HDL on melatonin $6 \mathrm{mg} /$ day for 12 weeks group compared to placebo group. This concluded that melatonin can be a nutritional supplement that is beneficial for the improvement of metabolic syndrome components. ${ }^{28}$ The effect of melatonin administration in metabolic syndrome animal model showed an improvement in NO (Nitric Oxide) end products (NOx) and had a beneficial effect on the NOADMA (Asymmetric dimethylarginine) pathway as reported by Pasaoglu et al,2020. ${ }^{29}$

\section{Melatonin Role on Diabetes Mellitus Complication}

The condition of hyperglycemia causes excessive production of ROS, damaging oxidative proteins and lipid peroxidases, associated to pathogenesis of DM complication. Here are studies related to melatonin in DM complication..

\section{Melatonin Role on Diabetic Eyes Diseases}

Khorsand et al.,2016 proved melatonin preventing action in formation of cataract due to DM in mice. A cataract is characterized by rubbing eye lens, making vision blurred. Diabetic cataract pathogenesis is due to hyperglycemia, causing lipid peroxidase and glucose metabolic imbalance. Glucose metabolic imbalance involves a polyol pathway which requires two enzymes: Aldose Reductase (AR), and sorbitol dehydrogenase (SDH ).30 AR is an enzyme catalyzing process of forming glucose to sorbitol. Sorbitol excessive accumulation causes diabetes cataracts. Glutathione (GSH) is an endogenous antioxidant located on lens.31 The results showed levels of $\mathrm{AR}, \mathrm{SDH}$, and sorbitol of DM group were significantly higher, meanwhile MDA level and GSH were lower. The melatonin decreased activity of the enzymes. Thus, melatonin has an effect of increasing antioxidant parameters and has an inhibitory effect on $\mathrm{AR}$ and SDH to reduce sorbitol production so that the accumulation of these products on lens are prevented.

Study by Jiang T et al. 2015 successfully explored melatonin role on diabetic mouse retina. A previous theory also stated melatonin capability to strengthen cellular antioxidant role through phosphoinositide-3 kinase (PI3K)/Akt-Nrf2 SP and inhibitting production of proinflammatory cytokines.32 Oxidative stress mechanism is measured by GSH and glutamate cysteine ligase (GCL) which is a catalytic enzyme in GSH biosynthesis. GCL enzyme regulation requires a key transcription factor, Nrf2. NRF2 is regulated by a number of SPs, including PI3K/Akt.33 This study indicates degresion levels of retinal GSH, Akt phosphorylation and Nrf2 within group of Streptozotocin-induced (STZ) mice. However, after melatonin administration given, it can stimulate Akt phosphorylation in vivo to increase activity of Nrf2 functioning in GCL regulation. This process results to increase GSH production as an antioxidant and reduce retinal injury from oxidative stress through PI3K/Akt-Nrf2 signaling pathway. Other indicators measured were proinflammatory cytokines such as TNF- $\alpha$, IL-1 $\beta$ and inducible nitric oxide synthase (iNOS). Melatonin reduces those proinflammatory 
Table 1. Melatonin Role in Diabetes Mellitus

\begin{tabular}{|c|c|c|c|c|c|c|}
\hline No & $\begin{array}{c}\text { Author } \\
\mathrm{s}\end{array}$ & Sample & $\begin{array}{c}\text { Number of } \\
\text { samples }\end{array}$ & $\begin{array}{l}\text { Melatonin } \\
\text { role in DM }\end{array}$ & Results & Parameters \\
\hline 1 & $\begin{array}{l}\text { Kdziora } \\
\text { et al, } \\
2009^{23}\end{array}$ & Human & $\begin{array}{l}\text { NIDDM } \\
(15) / \text { Con } \\
(15)\end{array}$ & $\begin{array}{l}\text { Incidence } \\
\text { of DM }\end{array}$ & $\begin{array}{l}\text { MA increased SOD-1 activity, } \\
\text { and reduction of MDA level } \\
\text { and ceruloplasmin (Cp) } \\
\text { oxidase activity significantly }\end{array}$ & $\begin{array}{l}\text { Erythrocytic } \\
\text { malondialdehyde (MDA), } \\
\text { SOD-1 activity and } \\
\text { Ceruloplasmin (Cp) oxidase } \\
\text { activity. }\end{array}$ \\
\hline 2 & $\begin{array}{l}\text { Jimenéz } \\
\text { et al, } \\
2014^{8}\end{array}$ & $\begin{array}{l}\text { Zucker } \\
\text { diabetic } \\
\text { fatty (ZDF) } \\
\text { rats }\end{array}$ & $\begin{array}{l}\mathrm{DM}(8) / \\
\text { Con (8) }\end{array}$ & $\begin{array}{l}\text { Incidence } \\
\text { of DM }\end{array}$ & 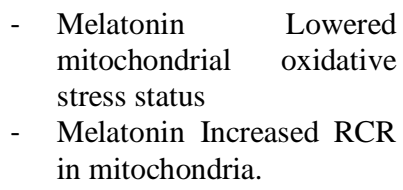 & $\begin{array}{l}\text { Nitrite (NO) and } \\
\text { Superoxide dismutase } \\
\text { (SOD) }\end{array}$ \\
\hline 3 & $\begin{array}{l}\text { Ozkanl } \\
\text { ar et al, } \\
2016^{9}\end{array}$ & $\begin{array}{l}\text { Sprague- } \\
\text { Dawley } \\
\text { rats }\end{array}$ & $\begin{array}{l}\mathrm{Con}(9) / \mathrm{DM} \\
(9) / \mathrm{Mel}(9) / \\
\mathrm{DM}+\mathrm{Mel}\end{array}$ & $\begin{array}{l}\text { Incidence } \\
\text { of DM }\end{array}$ & $\begin{array}{l}\text { MA controlled immune system } \\
\text { regulation and inhibit } \\
\text { production } \\
\text { proinflammatory cytokines } \\
\text { and tissue mast cell } \\
\text { accumulation. Decreased } \\
\text { WBC, Monocyte, and } \\
\text { Neutrophil counts }\end{array}$ & $\begin{array}{l}\text { - Serum interleukin } 1 \beta \text { (IL- } \\
1 \beta \text { ) and Tumor necrosis } \\
\text { factor alpha (TNF- } \alpha \text { ) } \\
\text { - hematologic analysis, } \\
\text { complete blood counts, } \\
\text { white blood cells (WBC), } \\
\text { neutrophils (Neut), } \\
\text { lymphocytes (Lym), and } \\
\text { monocytes (Mon) } \\
\text { - Mast Cell activation }\end{array}$ \\
\hline
\end{tabular}

cytokines in retina. As a conclusion, melatonin attenuates inflammation process and oxidative stress in retina causing diabetic retinopathy.

\section{Melatonin Role on Diabetic Cardiac Diseases}

$\mathrm{DM}$ causes myocardial ischemia-reperfusion (MI/R) injury, an injury because of reperfusion process (blood flow return) due to previously occurred myocardium

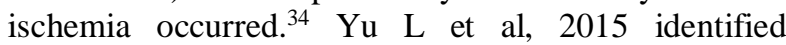
melatonin role in preventing MI/R injury through Silent information regulator 1 (SIRT1) ${ }^{35}$ Degression of SIRT1 can be found in diabetic experimental animals. ${ }^{36}$ SIRT1 is a protein deacetylase functioning in several cellular functions such as cell cycle regulation, apoptosis, aging, and DNA repair. ${ }^{37}$ Mice suffering DM had more severe cardiac damage caused by an increase in myocardial oxidative stress and endoplasmic reticulum (ER) stress. The DM group suffering $M I / R$ injury had higher myocardial and MDA superoxide levels. The DM+MI/R injury group had significantly lower SOD levels. This confirms that hyperglycemic conditions exacerbate oxidative stress in cardiac tissue of the mice. Melatonin given orally by dilution for 1 week at dose $20 \mathrm{mg} / \mathrm{kg} /$ day improve recovery of cardiac post-MI/R injury. Melatonin significantly reduces oxidative stress by reducing superoxide generation and MDA levels and also increasing SOD. In conclusion, the MA significantly increases expression of SIRT1, decreases stress ER. MI/R injury process involves MitogenActivated Protein Kinase (MAPK), Nrf-2-HO-1, and cGMP-PKGI $\alpha$ as evidenced by Yu L et al, 2017. Melatonin is capable of carrying out its function by activating membrane receptors and regulating intracellular signalling pathaways (SPs) such as Cyclic Guanosine Monophosphate (cGMP)-protein kinase G (PKG) signaling.38,39 cGMP-PKG interacts with a descriptive factors, Nuclear factor-erythroid-2 (NF-E2)related factor 2(Nrf-2) regulating expression of antioxidant genes and cytoprotective enzymes on cardiovascular system. Furthermore, cGMP-PKG modulates cascade of MAPK to induce cardiomyocyte cell apoptosis, resulting in MI/R injury. ${ }^{40,41}$ This study proved that giving melatonin increased cGMP-PKG, seen from increasing PKGI $\alpha$ expression, VASP phosphorylation, and cGMP in diabetic mice. Melatonin's role is to stimulate reduction of oxidative stress in myocardial cells.

Diabetic cardiomyopathy (DC) is a diabetic complication with myocardial structure damage and its function without being caused by the presence of other cardiac diseases such as hypertension, coronary heart or heart valve disorders. ${ }^{42}$ According to American Heart Association, 2013, clinical condition of DC was in the form of ventricular dysfunction caused by ventricular hypertrophy, fibrosis, cardiomyocyte death and disorders of cell signaling. Cardiomyocyte death pathogenesis are mitochondrial oxidative stress and imbalance of endoplasmic reticulum/ ER-Calcium. ${ }^{43,44}$ Hyperglycemic in DM patients may activate Spleen Tyrosine Kinase (Syk), a non-receptor protein kinase functioning in signal transduction that causing DC. Activating Syk reduces activity of mitochondrial complex I (COX-1) to stimulate production of cellular ROS and oxidative stress occurs. Zhou $\mathrm{H}$ et al., 2018 analyzed melatonin role having an antioxidant effect in incidence of DC by focusing on the Syk pathway. ${ }^{15}$ The syk pthwys inhibition by melatonin in this study involves a degression in anti-inflammatory cytokines: TGF $\beta$, IL6, TNF $\alpha$, MMP9, MCP1, IL8 and collagen III. This prevents cardiac inflammation to cause DC.

Another melatonin cellular function is mediating mitochondrial fission process by assistance of dynaminrelated protein 1 (Drp1). The mitochondrial fusion improves mitochondrial function and ATP production. Conversely, mitochondrial fission is detrimental because it reduces mitochondrial function and increases ROS. ${ }^{45,46}$ Increased mitochondrial fission in DM patients underlies the occurrence of myocardial contractile 
Table 2. Melatonin Role on Diabetes Mellitus Complication

\begin{tabular}{|c|c|c|c|c|c|c|}
\hline No & Author & Sample & $\begin{array}{c}\text { Number of } \\
\text { samples }\end{array}$ & $\begin{array}{l}\text { Melatonin } \\
\text { role in DM }\end{array}$ & Results & Parameters \\
\hline \multicolumn{7}{|c|}{ Melatonin Role on Diabetic Eyes Diseases } \\
\hline 1 & $\begin{array}{l}\text { Khorsand } \\
\text { et al, } \\
2016^{10}\end{array}$ & $\begin{array}{l}\text { Sprague- } \\
\text { Dawely } \\
\text { Diabetic rats }\end{array}$ & $\begin{array}{l}\mathrm{DM}(34) / \\
\text { Con }(34)\end{array}$ & $\begin{array}{l}\text { Diabetic } \\
\text { cataract }\end{array}$ & $\begin{array}{l}\text { MA delayed cataract progression } \\
\text { by increasing antioxidant effect } \\
\text { and and inhibiting Aldose }\end{array}$ & $\begin{array}{l}\text { Glutathione (GSH), Aldose } \\
\text { Reductase (AR), sorbitol } \\
\text { dehydrogenase (SDH) }\end{array}$ \\
\hline & 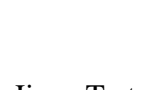 & S & & $1 \cdot 1$. & $\begin{array}{l}\text { Reductase (AR), sorbitol } \\
\text { dehydrogenase (SDH) enzyme. }\end{array}$ & - Plasma malondialdhyde \\
\hline 2 & $\begin{array}{l}\text { Jiang T et } \\
\text { al, } 2016^{11}\end{array}$ & $\begin{array}{l}\text { Sprague- } \\
\text { Dawley rats }\end{array}$ & NA & $\begin{array}{l}\text { diabetic } \\
\text { retinopathy }\end{array}$ & $\begin{array}{l}\text { MA prevents degression in } \\
\text { glutathione and Glutamat } \\
\text { cysteine ligase (GCL) while } \\
\text { maintaining Nrf2 in the nucleus, } \\
\text { stimulating Akt phosphorylation } \\
\text { and inhibiting proinflammatory } \\
\text { cytokines through the NF-kB } \\
\text { pathway. }\end{array}$ & $\begin{array}{ll}\text { - } & \text { Glutathione and CGL } \\
\text { - } & \text { interleukin } 1 \beta, \text { TNF- } \alpha \text {, and } \\
& \text { i NOS }\end{array}$ \\
\hline \multicolumn{7}{|c|}{ Melatonin Role on Diabetic Cardiac Diseases } \\
\hline 1 & $\begin{array}{l}\text { Yu L, } \\
2015^{35}\end{array}$ & $\begin{array}{l}\text { High-fat diet- } \\
\text { fed } \\
\text { streptozotocin } \\
\text { (HFD-STZ) rat }\end{array}$ & $\begin{array}{l}\text { DM (150)/ } \\
\text { Con (60) }\end{array}$ & $\begin{array}{l}\text { Myocardial } \\
\text { ischemia- } \\
\text { reperfusion } \\
\text { (MI/R) } \\
\text { injury }\end{array}$ & $\begin{array}{l}\text { MA reduced MI/R injury damage } \\
\text { and improved cardiac function by } \\
\text { up-regulating SIRT } 1 \text { expression, } \\
\text { reducing oxidative damage, and } \\
\text { suppressing PERK/eIF2a/ATF4. }\end{array}$ & $\begin{array}{l}\text { - Silent information regulator } 1 \\
\text { (SIRT1) } \\
\text { - PERK/eIF2a/ ATF4-mediated } \\
\text { ER stress } \\
\text { - myocardial superoxide } \\
\text { generation } \\
\text { - Malondialdehyde (MDA), } \\
\text { - superoxide dismutase (SOD) }\end{array}$ \\
\hline 2 & $\begin{array}{l}\text { Mingming } \\
Z \text { et al, } \\
2017^{12}\end{array}$ & $\begin{array}{l}\text { Mst1 } \\
\text { transgenic } \\
\text { (Mst1 Tg) and } \\
\text { Mst1 knockout } \\
\text { (Mst1-/-) } \\
\text { mice }\end{array}$ & $\begin{array}{l}\text { Con }(20) / \text { Con } \\
+\mathrm{Mel}(20) / \mathrm{D} \\
\mathrm{M}(20) / \mathrm{DM}+ \\
\mathrm{Mel} \\
(20)\end{array}$ & $\begin{array}{l}\text { Diabetic } \\
\text { cardiomyop } \\
\text { athy }\end{array}$ & $\begin{array}{l}\text { Melatonin alleviates cardiac } \\
\text { remodeling and dysfunction in } \\
\text { diabetic cardiomyopathy by up- } \\
\text { regulating autophagy, limiting } \\
\text { apoptosis and modulating } \\
\text { mitochondrial integrity and } \\
\text { biogenesis through Mst1/Sirt3 }\end{array}$ & $\begin{array}{l}\text { - ROS production and } \\
\text { MnSOD activity } \\
\text { p62 expression, beclin1, } \\
\text { Atg5 and LC3-II } \\
\text { expression }\end{array}$ \\
\hline 3 & $\begin{array}{l}\text { Yu L, et } \\
\text { al, } 2017^{16}\end{array}$ & $\begin{array}{l}\text { diabetic } \\
\text { Sprague- } \\
\text { Dawley rats }\end{array}$ & $\begin{array}{l}\text { Con }(12) / \\
\text { DM(12)/DM } \\
+ \text { Sham op } \\
(24) / \text { DM+ } \\
\text { MI/R+Vehic } \\
\text { le treatment } \\
\text { (24)/DM+ } \\
\text { MI/R+Mel } \\
(24) / \text { DM+ } \\
\text { MI/R+Mel+ } \\
\text { KT5823(24)/ } \\
\text { DM+KT582 } \\
3(12) / \\
\text { DM+MEL } \\
(12)\end{array}$ & $\begin{array}{l}\text { Myocardial } \\
\text { ischemia- } \\
\text { reperfusion } \\
\text { (MI/R) } \\
\text { injury }\end{array}$ & $\begin{array}{l}\text { MA reduced myocardial } \\
\text { apoptosis, oxidative stress and } \\
\text { preserving cardiac function by } \\
\text { modulating cGMP-PKG, Nrf-2- } \\
\text { HO-1 and MAPK }\end{array}$ & $\begin{array}{ll}\text { - } & \text { PKGI } \alpha \text { expression } \\
\text { - } & \text { VASP phosphorylation } \\
\text { - } & \text { c GMP level } \\
\text { - } & \text { SOD ,MDA }\end{array}$ \\
\hline 4 & $\begin{array}{l}\text { Zhou H et } \\
\text { al,2018 }\end{array}$ & rats & 6 & $\begin{array}{l}\text { Diabetic } \\
\text { cardiomyop } \\
\text { athy }\end{array}$ & $\begin{array}{l}\text { MA delayed DC by suppressing } \\
\text { spleen tyrosine kinase (Syk). }\end{array}$ & $\begin{array}{ll}\text { - } & \text { Antioxidant activity (SOD, } \\
\text { GSH,GPx) } \\
\text { - } & \text { Spleen tyrosine kinase } \\
\text { (Syk) activity } \\
\text { - } & \text { Sitokin proinflamatori } \\
\text { (TGF } \beta \text {, IL6, TNF } \alpha, \text { MMP9, } \\
\text { MCP1, IL8 ) and collagen } \\
\text { III }\end{array}$ \\
\hline 5 & $\begin{array}{l}\text { Ding M, } \\
\text { et al } \\
2018^{47}\end{array}$ & Diabetic mice & $\begin{array}{l}\mathrm{DM} \\
(8) / \mathrm{DM}+\mathrm{Mel} \\
(8) / \mathrm{SIRT}^{-/-} \\
\mathrm{DM}(8) / \\
\mathrm{SIRT}^{-/-} \mathrm{DM}+ \\
\operatorname{Mel}(8)\end{array}$ & $\begin{array}{l}\text { Myocardial } \\
\text { contractile } \\
\text { dysfunction }\end{array}$ & $\begin{array}{l}\text { Melatonin prevents } \\
\text { mitochondrial fission by } \\
\text { increasing expression of } \\
\text { SIRT1 and PGC-1 } \alpha \\
\text { Melatonin improved } \\
\text { mitochondrial function and } \\
\text { cardiac function by } \\
\text { decreasing } \text { dynamin- } \\
\text { related protein } 1 \text { (Drp1) } \\
\text { expression, oxidative } \\
\text { stress, mitochondrial } \\
\text { fragmentation, } \\
\text { cardiomyocyte apoptosis. }\end{array}$ & 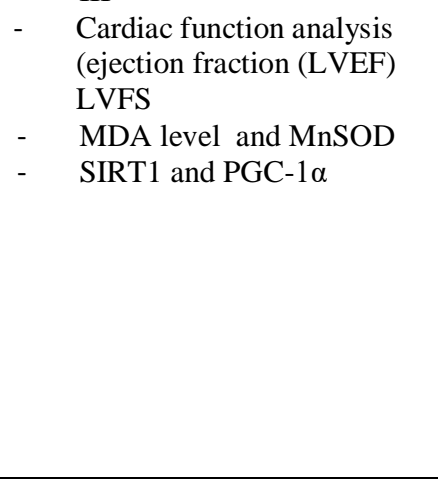 \\
\hline
\end{tabular}


Table 2. Continuous.

\begin{tabular}{|c|c|c|c|c|c|c|}
\hline \multicolumn{7}{|c|}{ Melatonin Role on Diabetic Male Reproductive Diseases } \\
\hline 1 & $\begin{array}{l}\text { Qiu XF et } \\
\mathrm{al}, 2012^{14}\end{array}$ & Rats & $\begin{array}{l}\text { Con (10)/DM } \\
\text { (11)/ Mel (11) }\end{array}$ & $\begin{array}{l}\text { erectile } \\
\text { dysfunction }\end{array}$ & $\begin{array}{l}\text { The chronic MA } \\
\text { increased circulating } \\
\text { EPC levels, diminished } \\
\text { oxidative stress and } \\
\text { restored endothelium } \\
\text { structur of corpus } \\
\text { cavernosum. }\end{array}$ & $\begin{array}{l}\text { - Malondialdehyde (MDA), } \\
\text { superoxide dismutase (SOD) } \\
\text { - endothelial progenitor cells } \\
\text { (EPCs) by the percentage of } \\
\text { CD34 } / \mathrm{KDR}^{+} \mathrm{EPC} \text { cells in the } \\
\text { peripheral blood }\end{array}$ \\
\hline 2 & $\begin{array}{l}\text { Zhang JL et } \\
\text { al, } 2018^{13}\end{array}$ & $\begin{array}{l}\text { Sprague- } \\
\text { Dawley } \\
\text { rats }\end{array}$ & $\begin{array}{l}\text { Con (12)/ DM } \\
\text { (12)/ Mel (13) }\end{array}$ & $\begin{array}{l}\text { erectile } \\
\text { dysfunction } \\
\text { (ED) }\end{array}$ & $\begin{array}{l}\text { MA improved erectile } \\
\text { function and ameliorate } \\
\text { neuropathy by inhibiting } \\
\text { p38MAPK pathway }\end{array}$ & $\begin{array}{ll}\text { - } & \text { Malondialdehyde (MDA), } \\
& \text { superoxide dismutase (SOD) } \\
\text { - } & \text { n-Nos expression in DPN and } \\
\text { MPG } & \\
\text { - } & \text { p38 and p-p38 levels } \\
\text { (p38MAPK SP) }\end{array}$ \\
\hline
\end{tabular}

Melatonin in DM rats decreased expression of Drp1, reducing oxidative stress, mitochondrial fragmentation and cardiomyocyte apoptosis and resulting in amelioration of mitochondrial and cardiac functions. ${ }^{47}$ Melatonin slows cardiac dysfunction by preventing mitochondrial fission through SIRT1-PGC1 $\alpha$ pathway associated with Drp1 expression. The role of melatonin in heart disease also occurs in controlling blood pressure. Results from studies using systematic reviews and randomized controlled trials show that melatonin supplementation has been shown to significantly reduce systolic blood pressure (SBP) and diastolic blood pressure (DBP). ${ }^{48}$ In addition, supplementation of melatonin is also useful in preventing atherosclerosis as reported by Li HY et al, 2019. ${ }^{49}$ This study proves the role of melatonin as an anti-inflammatory and antiproliferative involving VCAM-1 mRNA and TNF- $\alpha$.

\section{Melatonin Role on Diabetic Male Reproductive Diseases}

Diabetic neuropathy on male reproductive system involving neurovascular disorders is erectile dysfunction or impotence by inability to achieve or maintain an erection. Normally, Nitric oxide (NO) from penile nitrergic neurons and vascular endothelium stimulate production of cyclic guanosine monophosphate (cGMP), causing relaxation of corpus cavernosum and increases blood supply. DM patients have neurovascular and neuronal nitric synthase (nNOS) activity disorder, affecting relaxation function of corpus cavernosum. In addition, condition of hyperglycemia in DM also stimulates free radicals agents to negatively affect nerve cells, smooth muscle, and endothelial function. Antioxidant effect of melatonin manages erectile dysfunction due to excessive amounts of free radicals. Zhang JL et al, 2018 analyzed neuroprotective effects of melatonin in erectile dysfunction. ${ }^{13}$ The study, showed that rats given melatonin significantly restoring erectile dysfunction condition. An increased SOD activity and significantly decreased of MDA was found in Melatonin group, proving that Melatonin relieves oxidative stress. Melatonin mechanism in repairing erectile dysfunction appears to be through p38 mitogen-activated protein kinase (p38MAPK) pathway. P38MAPK is a MAPK superfamily, activable due to oxidative stress, hyperglycemia, and inflammation. Activating MAPK triggers diabetic neuropathy. The findings showed p-p38 levels after MA was significantly decreased, proving that melatonin inhibits (p38MAPK) pathways so erectile dysfunction can be prevented.

Erectile dysfunction mechanism (ED) is actually due to multifactorial risk. Several studies identified incidence of ED in relation to degression of Endothelial progenitor cells (EPCs). ${ }^{14,50} \mathrm{EPC}$ is a progenitor cell functioning in neovascularization process and re-endothelialization to maintain endothelial integrity. The erection process requires excellent endothelial function and structure as well as smooth muscle of corpus cavernosum. So if there is a degression in EPC it disrupts a man's erection. EPC reduction is triggered by the presence of oxidative stress interfering EPC mobilization process from bone marrow to peripheral circulation. Qiu XF et al, 2012 successfully identified role of longterm Melatonin in reducing oxidative stress and triggering EPC mobilization to prevent ED events. However, 8 weeks of MA showed an increase in endothelial density. This showed Melatonin role in restoring corpus cavernosum endothelium. ${ }^{14}$ Thus, long-term MA reduces oxidative stress so that EPC mobilization increases and improves endothelial structure of corpus cavernosum functioning in normal erection process.

\section{CONCLUSION}

Melatonin prevents various DM complication on eyes, cardiac, and male reproductive organs through antioxidant effects and proinflammatory inhibition. The pathways are PI3K/Akt-Nrf2,cGMP-PKG, Nrf-2-HO-1 and MAPK, Syk, p38MAPK pathway etc. This review provides various melatonin roles in diabetes and complications to present a promising new breakthrough on DM treatment. It is necessary to explore Melatonin role in other DM complications. Most of this research is on experimental animals, thus clinical trial studies should be carried out in humans to make melatonin a promising therapeutic or prevention for diabetes and its complications in the future.

\section{ACKNOWLEDGMENT}

The research is funded by the Directorate of Research and Community Services, Directorate General of Research and Development, Ministry of Research, Technology, and Higher Education, Republic of Indonesia, under the research scheme of Penelitian Kerjasama Antar Perguruan Tinggi, 2019. 


\section{REFERENCES}

1. World Health Organization. Global status report on noncommunicable diseases 2014. 2014.

2. Ministry of Health R of I. Basic health research 2013. 2014.

3. Central Java Health Office. Health profile of Central Java province 2017. Central Java Health Office. Semarang; 2018.

4. Peschke E, Frese T, Chankiewitz E, Peschke D, Preiss U, Schneyer U, et al. Diabetic Goto Kakizaki rats as well as type 2 diabetic patients show a decreased diurnal serum melatonin level and an increased pancreatic melatonin-receptor status. J Pineal Res. 2006;40(2):13543.Doi:10.1111/j.1600-079X.2005.00287.x

5. Ramracheya RD, Muller DS, Squires PE, Brereton H, Sugden D, Huang GC, et al. Function and expression of melatonin receptors on human pancreatic islets. J Pineal Res. 2008;44(3):2739.Doi:10.1111/j.1600-079X.2007.00523.x.

6. Peschke E. Melatonin, endocrine pancreas and diabetes. J Pineal Res. 2008;44(1):26-40. Doi:10.1111/j.1600-079X.2007.00519.x.

7. Peschke E, Mühlbauer E. New evidence for a role of melatonin in glucose regulation. Best Pr Res Clin Endocrinol Metab. 2010;24(5):829-41. Doi:10.1016/j.beem.2010.09.001.

8. Jimenéz AA, Fernández-Vázquez G, Mohammad ASM, Reiter RJ, Agil A. Melatonin improves mitochondrial function in inguinal white adipose tissue of Zücker diabetic fatty rats. J Pineal Res. 2014;57(1):103-9. Doi:10.1111/jpi.12147.

9. Ozkanlar S, Kara A, Sengul E, Simsek N, Karadeniz A, Kurt N. Melatonin modulates the immune system response and inflammation in diabetic rats experimentally-induced by Alloxan. Horm Metab Res. 2015;48(2):137-44. DOI: http://dx.doi.org/ 10.1055/s-0035-1548937 .

10. Khorsand M, Akmali M, Sharzad S, Beheshtitabar M. Melatonin reduces cataract formation and aldose reductase activity in lenses of streptozotocin-induced diabetic rat. Iran J Med Sci. 2016;41(4):305-13.

11. Jiang T, Chang Q, Cai J, Fan J, Zhang X, Xu G. Protective effects of melatonin on retinal inflammation and oxidative stress in experimental diabetic retinopathy. Oxid Med Cell Longev. 2016;2016:14-7. Doi.org/10.1155/2016/3528274.

12. Mingming Z, Jie L, Shanjie W, Zheng C, Jianqiang $\mathrm{H}$, Tingting $\mathrm{W}$,et al. Melatonin protects against diabetic cardiomyopathy through Mst1/Sirt3 signaling. J Pineal Res. 2017;63(2):1-26. Doi: 10.1111/jpi.12418.

13. Zhang J lei, Hui Y, Zhou F, Hou JQ. Neuroprotective effects of melatonin on erectile dysfunction in streptozotocin-induced diabetic rats. nt Urol Nephrol. 2018;50(11):198118.Doi.org/10.1007/s11255-018-1989-4.

14. Qiu XF, Li XX, Chen Y, Lin HC, Yu W, Wang R, et al. Mobilisation of endothelial progenitor cells: One of the possible mechanisms involved in the chronic administration of melatonin preventing erectile dysfunction in diabetic rats. Asian J Androl. 2012;14(3):481-6.Doi:10.1038/aja.2011.161.
15. Zhou H, Yue Y, Wang J, Ma Q, Chen Y. Melatonin therapy for diabetic cardiomyopathy: A mechanism involving Syk-mitochondrial complex I-SERCA pathway. Cell Signal. 2018;47:88100.Doi:10.1016/j.cellsig.2018.03.012.

16. Yu L, Di WC, Dong X, Li Z, Zhang Y, Xue XD, et al. Melatonin protects diabetic heart against ischemia-reperfusion injury, role of membrane receptor-dependent cGMP-PKG activation. Biochim Biophys Acta, Mol Basis Dis. 2018;1864(2):56378.Doi:10.1016/j.bbadis.2017.11.023 .

17. Kaczor BT. An overview of melatonin and breast cancer. Nat Med J. 2010;2(February):1-5.

18. Ritzel RA, Butler AE, Rizza RA, Veldhuis JD, Butler PC. Relationship between $\beta$-cell mass and fasting blood glucose concentration in humans. Diabetes Care. 2006;29(3):7178.Doi.org/10.2337/diacare.29.03.06.dc05-1538.

19. Brand MD, Nicholls DG. Assessing mitochondrial dysfunction in cells. Biochem J. 2011;435(2):297312. Doi:10.1042/BJ20110162

20. Zavodnik IB, Lapshina EA, Cheshchevik VT, Dremza IK, Kujawa J, Zabrodskaya S V., et al. Melatonin and succinate reduce rat liver mitochondrial dysfunction in diabetes. J Physiol Pharmacol. 2011;62(4):421-7.

21. González MA, del Carmen CM, Millen N, Mahieu ST. Role of melatonin in the oxidative damage prevention at different times of hepatic regeneration. Cell Biochem Funct. 2012;30(8):701-8 .Doi: 10.1002/cbf.2855

22. Yang Y, Duan W, Jin Z, Yi W, Yan J, Zhang S, et al. JAK2/STAT3 activation by melatonin attenuates the mitochondrial oxidative damage induced by myocardial ischemia/reperfusion injury. J Pineal Res. 2013;55(3):275-86.Doi:10.1111/jpi.12070.

23. Kdziora KK, Szewczyk GK, Kozakiewicz M, Pawluk H, Czuczejko J, Kornatowski T, et al. Melatonin improves oxidative stress parameters measured in the blood of elderly type 2 diabetic patients. J Pineal Res. 2009;46(3):333-7. Doi:10.1111/j.1600-079X.2009.00666.X.

24. Gil L, Siems W, Mazurek B, Gross J, Schroeder P, Voss P, et al. Age-associated analysis of oxidative stress parameters in human plasma and erythrocytes. Free Radic Res. 2006;40(5):495-505.Doi: 10.1080/10715760600592962.

25. Tie L, Li XJ, Wang X, Channon KM, Chen AF. Endothelium-specific GTP cyclohydrolase I overexpression accelerates refractory wound healing by suppressing oxidative stress in diabetes. Am J Physiol Endocrinol Metab. 2009;296(6):142319.Doi:10.1152/ajpendo.00150.2009.

26. Ozcelik IŞ. Beneficial effects of melatonin on oxidative damage observed during whole blood storage. J Hematol Oncol. 2014;24(2):89-96.Doi: 10.4999/uhod.13089.

27. Carrillo VA, Lardone PJ, Álvarez ŚN, Rodrĩguez RA, Guerrero JM. Melatonin: buffering the immune system. Int J Mol Sci. 2013;14(4):863883.Doi:10.3390/ijms 14048638. 
28. Bahrami M, Cheraghpour M, Jafarirad S, Alavinejad P, Cheraghian B. The role of melatonin supplement in metabolic syndrome: A randomized double blind clinical trial. Nutr Food Sci. 2019;49(5):965-77.Doi:10.1108/NFS-01-20190018.

29. Pasaoglu OT, Bircan FS, Topal T, Turkozkan N. Positive effects of melatonin on renal nitric oxideasymmetric dimethylarginine metabolism in fructose-fed rats. Metab Syndr Relat Disord. 2021;19(3):120-6.Doi: 10.1089/met.2020.0084.

30. Alwadani F, Saif M. The role and prevalence of polyol pathway and oxidative stress markers as risk factors for diabetic cataract in adult type-i diabetic and diabetic cataract Saudi patients. J Clin Exp Ophthalmol. 2016;7(3):1-8.Doi: 10.4172/21559570.1000558.

31. Zhang S, Chai FY, Yan H, Guo Y, Harding JJ. Effects of $\mathrm{N}$-acetylcysteine and glutathione ethyl ester drops on streptozotocin-induced diabetic cataract in rats. Mol Vis. 2008;14(April):862-70.

32. Jiang T, Chang Q, Zhao Z, Yan S, Wang L, Cai J, et al. Melatonin-mediated cytoprotection against hyperglycemic injury in müller cells. PLoS One. 2012;7(12):1-

8.Doi:10.1371/journal.pone.0050661.

33. Wang L, Chen Y, Sternberg P, Cai J. Essential roles of the PI3 kinase/Akt pathway in regulating Nrf2dependent antioxidant functions in the RPE. Investig Ophthalmol Vis Sci. 2008;49(4):16718.Doi: 10.1167/iovs.07-1099.

34. Frank A, Bonney M, Bonney S, Weitzel L, Ph D, Koeppen M, et al. Myocardial ischemia reperfusion injury - from basic science to clinical bedside. Semin Cardiothorac Vasc Anesth. 2012;16(3):12332.Doi: $10.1177 / 1089253211436350$.

35. Yu L, Liang H, Dong X, Zhao G, Jin Z, Zhai M, et al. Reduced silent information regulator 1 signaling exacerbates myocardial ischemia-reperfusion injury in type 2 diabetic rats and the protective effect of melatonin. J Pineal Res. 2015;59(3):37690.Doi:10.1111/jpi.12269.

36. Koka S, Aluri HS, Xi L, Lesnefsky EJ, Kukreja RC. Chronic inhibition of phosphodiesterase 5 with tadalafil attenuates mitochondrial dysfunction in type 2 diabetic hearts: Potential role of NO/SIRT1/PGC-1 $\alpha$ signaling. AM J PHYSIOLHEART C. 2014;306(11):155868.Doi:10.1152/ajpheart.00865.2013.

37. Yang Y, Duan W, Li Y, Jin Z, Yan J, Yu S, et al. Novel role of silent information regulator 1 in myocardial ischemia. Circulation. 2013;128(20):2232-40.Doi: 10.1161/CIRCULATIONAHA.113.002480.

38. Jockers R, Delagrange P, Dubocovich ML, Markus RP, Renault N, Tosini G, et al. Update on melatonin receptors. Br J Pharmacol . 2016;173(18):270225.Doi: 10.1111/bph.13536.

39. Tosini G, Owino S, Guillaume JL, Jockers R. Understanding melatonin receptor pharmacology: Latest insights from mouse models, and their relevance to human disease. BioEssays. 2014;36(8):778-87.Doi:10.1002/bies.201400017.
40. Wang J, Hu X, Jiang H. The Nrf-2/ARE-HO-1 axis: An important therapeutic approach for attenuating myocardial ischemia and reperfusion injury-induced cardiac remodeling. Int J Cardiol. 2015;184(1):2634.Doi.org/10.1016/j.ijcard.2015.02.073.

41. Das A, Salloum FN, Xi L, Rao YJ, Kukreja RC. ERK phosphorylation mediates sildenafil-induced myocardial protection against ischemia-reperfusion injury in mice. AM J PHYSIOL-HEART C. 2009;296(5):1236-

43.Doi:10.1152/ajpheart.00100.2009.

42. Jia G, Hill MA, Sowers JR. Diabetic cardiomyopathy: An update of mechanisms contributing to this clinical entity. Circ Res. 2018;122(4):624-38.

Doi: 10.1161/CIRCRESAHA.117.311586.

43. Van NJL, Bowen ME, Vogel H, Barna M, Attardi LD. The p53 family members have distinct roles during mammalian embryonic development. Cell Death Differ. 2017;24(4):5759.Doi:10.1038/cdd.2016.128.

44. Kozlov A V., Lancaster JR, Meszaros AT, Weidinger A. Mitochondria-meditated pathways of organ failure upon inflammation. Redox Biol. 2017;13(May):170-81

.Doi.org/10.1016/j.redox.2017.05.017.

45. Chiong M, Cartes-Saavedra B, Norambuena-Soto I, Mondaca-Ruff D, Morales PE, García-Miguel M, et al. Mitochondrial metabolism and the control of vascular smooth muscle cell proliferation. Front Cell Dev Biol. 2014;2(DEC):1-9.Doi: 10.3389/fcell.2014.00072

46. Ong SB, Hall AR, Hausenloy DJ. Mitochondrial dynamics in cardiovascular health and disease. Antioxid Redox Signal. 2013;19(4):400-14.Doi: 10.1089/ars.2012.4777.

47. Ding M, Feng N, Tang D, Feng J, Li Z, Jia M, et al. Melatonin prevents Drp1-mediated mitochondrial fission in diabetic hearts through SIRT1-PGC1 $\alpha$ pathway. Vol. 65, J. Pineal Res. 2018. 1-48.Doi: 10.1111/jpi.12491.

48. Akbari M, Ostadmohammadi V, Mirhosseini N, Lankarani KB, Tabrizi R, Keshtkaran Z, et al. The effects of melatonin supplementation on blood pressure in patients with metabolic disorders: a systematic review and meta-analysis of randomized controlled trials. J Hum Hypertens. 2019;33(3):2029.Doi.org/10.1038/s41371-019-0166-2.

49. Li HY, Leu YL, Wu YC, Wang SH. Melatonin inhibits in vitro smooth muscle cell inflammation and proliferation and atherosclerosis in apolipoprotein e-deficient mice. J Agric Food Chem. 2019;67(7):1889-901.Doi: 10.1021/acs.jafc.8b06217.

50. Esposito K, Ciotola M, Maiorino MI, Giugliano F, Autorino R, De Sio M, et al. Circulating $\mathrm{CD} 34+\mathrm{KDR}+$ endothelial progenitor cells correlate with erectile function and endothelial function in overweight men. J Sex Med. 2009;6(1):107-14.Doi: 10.1111/j.1743-6109.2008.01042.x. 